\title{
U na aproximación a la situación de los Agentes de Desarrollo Local (ADL) en el proceso de creación de nuevos polígonos industriales
}

\section{Ricard Calvo Palomares}

Universitat de València

\section{NOTA BIBLIOGRÁFICA}

RICARD CAL VO PALO MARES, Licenciado en Ciencias del Trabajo y Diplomado Graduado Social por la U niversitat de València. Es actualmente profesor asociado del Departamento de Sociología y Antropología Social de la U niversitat de València, desarrollando actividades docentes en diferentes asignaturas como, Regulación de Políticas de O cupación, Técnicas de Investigación Social, Medio Ambiente y Relaciones Laborales y Sociología del Trabajo y de las Relaciones Laborales. Su experiencia profesional en el sector privado alcanza aspectos tales como la consultoría, investigación y formación de recursos humanos, el desarrollo de planes de mejora, el asesoramiento jurídico-laboral, la negociación colectiva, la prevención de riesgos laborales, el desarrollo local, etc. Está actualmente en proceso de elaboración de su tesis donde su interés científico se centra en el análisis de las políticas activas de empleo, prestando especial atención a los Agentes de Desarrollo Local como instrumentos claves del proceso. Ha publicado artículos relacionados con la materia en revistas científicas como Lan H arremanak: Revista de Relaciones Laborales, Arxius: Revista de Ciències Socials, Trabajo, Aposta Digital, Cuadernos de Administración Local y Herramientas: Revista de Empleo y Formación.

\section{RESUMEN}

Un modelo de desarrollo local como el que tenemos implantado, aparecido a mediados de los años ochenta como respuesta a una situación de grave crisis económica y social, presenta hoy en día grandes carencias de tipo estructural, muchas de las cuales se han ido originando de facto con el transcurso de los años sin que nadie haya recogido el testigo de completar dichos 
vacíos. En esta nota destacamos una de ellas que consideramos muy visible en la actualidad: ante la floración continua de polígonos industriales -fuente teórica de empleo a nivel local- denotamos como el instrumento básico creado por el modelo de desarrollo local, los Agentes de Empleo y Desarrollo Local -AEDL o simplemente ADL- quedan apartados del proceso de su diseño y concepción, dedicando su labor profesional a tareas complementarias a las anteriores. Sobre la base de un estudio realizado en la comarca valenciana de La Ribera Alta, pretendemos poner de manifiesto esta situación y reflexionar sobre los principales factores que la causan.

PALABRAS CLAVE: ADL, Desarrollo local, Políticas de empleo, Agentes de Desarrollo Local, Empleo local.

\section{AN APPROACH TO THE STATUS OF THE LOCAL DEVELOPMENT (LDA) IN THE PROCESS OF CREATING A NEW INDUSTRIAL DIS- TRICTS}

\section{ABST RACT}

A local development model such as the one we have in place, appeared in the mid-eighties in response to a situation of serious economic and social crisis, today introduced major structural deficiencies, many of which have been de facto rise over the years without anyone has picked up the baton to complete these gaps. In this paper we emphasize one that we think is very visible at the moment: before flowering continuous industrial estates-theoretical source of local employment-denote the basic tool created by the model of local development agents and Employment Development Local -LDAE or simply LDA- are pushed aside in the process of design and concept, devoting their professional tasks complementary to the previous ones. Based on a survey conducted in the district of La Ribera Alta (Valencia), we want to highlight this situation and think about the major factors that cause.

KEYWO RDS: LDA, Local development Agency, Employment policies, Local development, Local employment.

\section{INTRODUCCIÓN}

Conforme a lo establecido en la Orden de 15 de julio de 1999 del Ministerio de Trabajo y Asuntos Sociales sobre "Fomento del Desarrollo Local e im- 
pulso de los proyectos y empresas calificadas como I +E"1, los Agentes de Empleo y Desarrollo Local -en adelante AEDL o ADL- se configuran como trabajadores de las corporaciones locales 0 entidades dependientes 0 vinculadas a una Administración local que tienen como misión principal colaborar en la promoción e implantación de las políticas activas de empleo relacionadas con la creación de actividad empresarial, desarrollándose dicha colaboración en el marco de actuación conjunta y acordada de la entidad contratante y el Instituto Nacional de Empleo².

Es por tanto el AEDL un instrumento al servicio del empleo a nivel local, que se enfrenta a un reto complejo, como es la mejora económica y social de un territorio en concreto ${ }^{3}$. El técnico ADL será el encargado de implementar, desarrollar y ejecutar los programas para el empleo generados de la aplicación de las políticas activas de empleo, y en su desarrollo sufrirá los efectos de una situación acrónica a su actividad, ya que su labor de intentar mejorar de alguna manera la misma en determinadas ocasiones será manifiestamente imposible.

La Unión Europea desde hace una década apuesta por el nivel local y lo reconoce como el escenario idóneo en el que conseguir los objetivos de mejora social pretendidos en el territorio. Se trata de una apuesta por lo próximo, por lo que la cercanía con la situación a mejorar y sobre la que se plantea una intervención, se convierte en el instrumento fundamental de trabajo. En

1 Modificada parcialmente por la Orden de 27 de diciembre de 1999, por la Orden 49/2005, de 14 de enero y recientemente por la orden TAS/ 360/ 2008, de 6 de febrero.

2 En la actualidad en aquellas Comunidades Autonómicas con las competencias transferidas en materia de empleo, son los órganos propios los encargados de gestionar estas subvenciones.

${ }^{3}$ Cabe citar que en el artículo 8 de la citada orden, se indican las funciones concretas a desarrollar por un ADL: 1. Prospección de recursos ociosos o infrautilizados, de proyectos empresariales de promoción económica local e iniciativas innovadoras para la generación de empleo en el ámbito local, identificando nuevas actividades económicas y posibles emprendedores; 2 . Difusión y estímulo de potenciales oportunidades de creación de actividad entre los desempleados, promotores y emprendedores, así como instituciones colaboradoras; 3. Acompañamiento técnico en la iniciación de proyectos empresariales para su consolidación en empresas, los proyectos generadores de nuevos empleos, asesorando e informando sobre la viabilidad técnica, económica y financiera y, en general, sobre los planes de lanzamiento de las empresas; 4. Apoyo a promotores de las empresas, una vez constituidas éstas, acompañando técnicamente a los mismos durante las primeras etapas de funcionamiento, mediante la aplicación de técnicas de consultoría en gestión empresarial y asistencia en los procesos formativos adecuados para coadyuvar a la buena marcha de las empresas creadas; y 5 . Cualesquiera otras que contribuyan a la promoción e implantación de políticas activas de empleo e impulse la creación de actividad empresarial. 
este sentido, la Estrategia Europea para el Empleo (EEE) establece las directrices y posibilita el inicio de una vía en la que los técnicos locales de empleo, pueden asumir un papel más activo todavía en su búsqueda de la mejora socioeconómica de la localidad, ya que les permite solicitar y defender en nombre propio los programas para el empleo sin tener que pasar por un filtro autonómico que hasta el momento suponía una limitación en la aplicación del proceso.

En este contexto la creación de suelo industrial parece ser una de las herramientas a emplear por parte del ADL en el desarrollo de su actividad. Se trata de un proceso "de moda" implantado en la actual existencia de los municipios, donde la concentración de empresas en espacios adecuados para las mismas con los servicios necesarios para la generación de unos efectos positivos para el empleo, se posiciona como primer aspecto de mejora para el futuro de la localidad. Dada la descripción realizada de la labor profesional del técnico ADL parece lógico pensar que él va a ser el encargado natural del desarrollo de los mismos, o al menos una de las piezas clave en el engranaje que pongan en funcionamiento dichos sistemas. Sin embargo la realidad nos va a indicar una situación bien distinta, estos técnicos para el empleo van a quedar apartados y aparcados de este proceso en el que su voz no va a ser tenida en cuenta, o al menos no de la manera que debería serlo.

\section{OBJETIVOS DEL ESTUDIO}

El estudio tuvo lugar entre los meses de abril y junio del año 2006. El objetivo general de nuestra investigación fue estudiar el papel desarrollado por los agentes de desarrollo local -ADL's- en la aplicación, desarrollo y ejecución de las políticas públicas de empleo en la comarca valenciana de La Ribera Alta desde la aparición de las primeras agencias hasta la actualidad.

Para poder alcanzar este objetivo genérico, nos planteamos otros de carácter específico y complementarios a éste, como los siguientes: analizar las características del entorno que rodea el agente en el desarrollo de su actividad; conocer el momento en el que son constituidas las diferentes agencias de la comarca y analizar los motivos que llevaron a los distintos municipios a solicitar la constitución de éstas; conocer las características básicas de la tarea desarrollada y los recursos humanos de que dispone; analizar las características propias del agente, y obtener un perfil de éste basado en variables como, la edad, el sexo, la antigüedad en el puesto de trabajo, la titulación académica, la formación complementaria en la materia, la experiencia previa y la relación 
laboral-contractual; conocer las líneas de trabajo desarrolladas por los ADL's de la comarca con exactitud, así como los programas de empleo desarrollados desde su constitución como tal, y analizar la evolución del tipo de programas de empleo desarrollados por los ADL's de La Ribera Alta en todo este tiempo, por años y por agencias; conocer los colectivos a los que se dirige, tipo de empresas con las que entra en contacto y características del mercado local de trabajo, analizar el grado de implicación social de la actividad profesional del técnico, y cuál es la principal orientación de ésta; y, por último analizar las características del servicio que preste el agente y los principales impedimentos con que se encuentra, y, el grado de implicación política municipal existente a los distintos municipios.

\section{LA HIPÓTESIS ESPECÍFICA DE ESTA NOTA}

Tras un estudio exploratorio inicial, surgió una línea de investigación materializada en la hipótesis central de esta nota de investigación, en la que planteamos la vinculación directa del trabajo desarrollado por los técnicos ADL's, con la consideración y la valoración del resto de departamentos de las Corporaciones locales, y más concretamente sobre la importancia asignada a su actividad. Fruto de ello, se continuó trabajando en este sentido, planteando una cuestión de fondo sobre la no participación de los técnicos locales de empleo en la creación de los polígonos industriales y sobre los factores que podían causar dicha situación.

\section{METODOLOGÍA Y POBLACIÓN DE REFERENCIA DE LA INVESTIGACIÓN}

Para desarrollar esta investigación se decidió emplear tres tipos de herramientas con la totalidad de técnicos -concretamente 26 técnicos correspondientes a las 18 agencias locales existentes- que estaban trabajando en dicho momento en las agencias de desarrollo del área geográfica de La Ribera Alta. La primera de las técnicas fue un cuestionario que se les mandó a los ADL's por correo electrónico, en el que se les pedía información básica respecto de su actividad y de los programas de empleo desarrollados hasta el momento 4 .

\footnotetext{
${ }^{4}$ Los contenidos del cuestionario remitido por correo electrónico anterior a la entrevista versaba sobre las siguientes informaciones: Fecha aproximada de inicio de la actividad de la agencia de desarrollo local; Número de recursos humanos disponibles de carácter estable en la agencia; Programas de empleo desarrollados hasta el momento; y Población activa y Número de habitantes de la población.
} 
En segundo lugar una entrevista personal con ellos, se optó por una entrevista semiestructurada donde hubieran establecidos unosítems básicos de trabajo común a todos, y que nos permitiera adaptarla a cada situación en concreto, y así poder profundizar en los aspectos más interesantes de cada uno de los $A D L$ ' s entrevistados ${ }^{5}$. Y en tercer lugar, se planteó la realización de un grupo de discusión entre los técnicos locales de empleo con un objetivo doble, por un lado presentar las conclusiones obtenidas hasta ese momento para que las validaran y por otro generar un pequeño debate entre ellos ${ }^{6}$.

Por lo que respecta a las técnicas de análisis aplicadas a los datos recogidos, hemos utilizado básicamente dos: unas de carácter cuantitativo, sobre todo para trabajar con los datos recogidos de los cuestionarios enviados, y por otra, dado que la entrevista y el grupo de discusión son herramientas de recogida de información de carácter cualitativo, hemos utilizado la técnica de análisis de contenidos de las mismas.

\section{LA OPERACIONALIZACIÓN DEL PROBLEMA}

Más allá de presentar algunos resultados descriptivos del estudio y como quiera que lo que se desea es analizar los motivos causantes de la situación en la que se encuentran los técnicos locales, presentamos a continuación un mapa conceptual -ver cuadro 1- en el que se exponen las principales variables del mismo, clasificadas según la función que cumplen en el proceso de investigación, independientes o dependientes, aspecto éste que es muy fácil de observar en la figura.

\footnotetext{
${ }^{5}$ Los contenidos básicos de la entrevista fueron los siguientes: breve descripción del mercado de trabajo de la localidad; Perfil del ADL (formación académica, complementaria y experiencia previa). ¿Cómo afrontas este trabajo?; ¿Cómo nace la agencia en esta localidad? Años de trabajo; Líneas básicas de trabajo de la agencia. O bjetivos de la misma; Actuaciones, acciones y programas desarrollados; Proceso seguido para la de detección de necesidades en la localidad; Aplicación de métodos para el seguimiento y medición de los efectos de los programas: percepción de las utilidades de la tarea desarrollada: ¿indicadores?; Principales dificultades con las que te encuentras en el desarrollo de tu tarea; Esfuerzo de la corporación municipal. Implicación política; Recursos disponibles (humanos y técnicos).

${ }^{6}$ Por lo que respecta al grupo de discusión se estructuró en tres rondas con turno rotatorio de palabra de los técnicos asistentes sobre los siguientes contenidos: 1. O pinión sobre las conclusiones expuestas, si había al guna con la que no estuvieran de acuerdo, o si añadirían ningún más; 2. ADL's, políticas de empleo y mejora social, ¿una realidad o una utopía?; y 3 . Principales retos de futuro del desarrollo local desde su perspectiva.
} 


\section{Cuadro 1. LASPRINCIPALES VARIABLES DEL ESTUDIO}
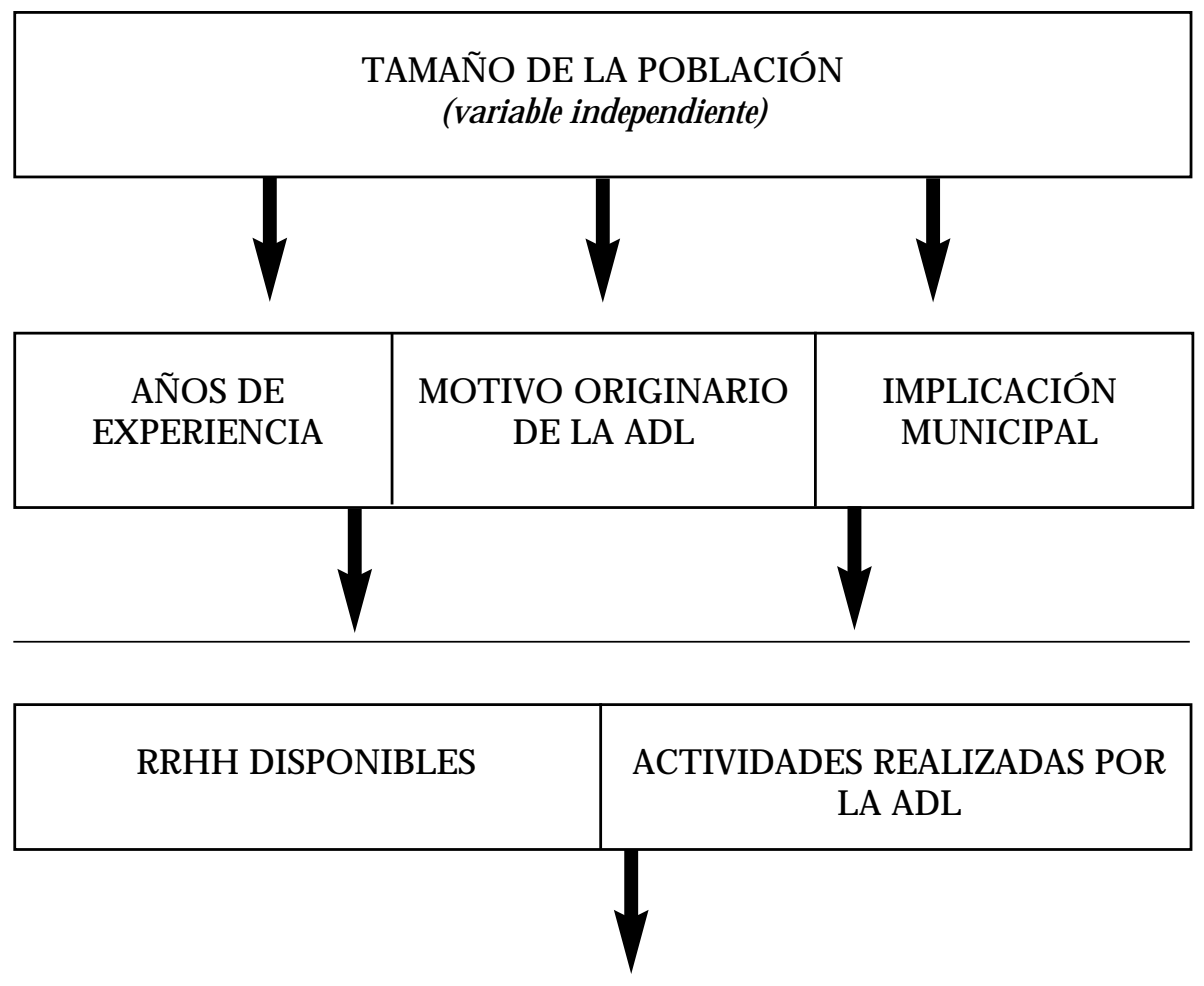

VALORACIÓN DEL TRABAJO

PARTICIPACIÓN O NO DE LOS ADL'SEN EL DISEÑO Y CREACIÓN DE POLÍGONOS INDUSTRIALES

Fuente- Elaboración propia 


\section{LOS DATOS MÁS RELEVANTES DEL UNIVERSO ESTUDIADO}

Importante es sin lugar a dudas contextualizar inicialmente los resultados del estudio realizado, para ello en la tabla 2 presentamos la información referente a la representatividad absoluta del modelo de desarrollo local en el ámbito territorial escogido. Más de un $80 \%$ de la población total de la comarca encuentra en su localidad la existencia de al menos un técnico ADL, lo que nos da muestras de la amplia cobertura del modelo en dicho territorio.

\section{Tabla 2 REPRESENTATIVDAD COMARCAL DE LOS MUNICIPIOS CON ADL PROPIA SOBRE LA POBLACIÓN TOTAL}

\begin{tabular}{|l|c|c|}
\hline & $\begin{array}{c}\text { TOTAL } \\
\text { HABITANTES }\end{array}$ & REPRESENTATIVIDAD \\
\hline TOTAL POBLACIÓN LA RIBERA ALTA & 210.637 & $100 \%$ \\
\hline $\begin{array}{l}\text { TOTAL POBLACIÓN MUNICIPIOS CON } \\
\text { ADL PROPIA }\end{array}$ & 172.206 & $81,75 \%$ \\
\hline
\end{tabular}

Fuente-Elaboración propia

Se trata, como hemos mencionado anteriormente, de un modelo originado en la década de los años ochenta y por tanto con más de veinte años de trabajo a sus espaldas. Pese a ello, la realidad del desarrollo local nos sitúa en un horizonte temporal entre los diez y quince años para la aparición de un contingente de ADL's considerado como importante. Desde el inicio de la década de los noventa el goteo de Agentes o de Agencias a nivel local, comarcal o supracomarcal ha sido continuo, siendo un factor clave en este sentido el tamaño de la población de destino de la misma.

Tabla 3. AÑOS DE EXPERIENCIA DE LASAGENCIAS

\begin{tabular}{|c|c|c|c|}
\hline & CANTIDAD & PORCENT AJE & MU NICIPIO S \\
\hline MÁS DE 10 AÑOS & 4 & $22,22 \%$ & $\begin{array}{c}\text { Alzira (42.543), Alberic (10.081), } \\
\text { Carlet (14.812), Algemesí (26.740) }\end{array}$ \\
\hline ENTRE 6 Y 10 AÑOS & 6 & $33,33 \%$ & $\begin{array}{l}\text { Turís (5.556), Carcaixent (21.299), } \\
\text { L'Alcúdia (10.838), Cárcer (2.040), } \\
\text { Benifaió (12.208), Tous (1.116) }\end{array}$ \\
\hline ENTRE 2 Y 5 AÑOS & 5 & $27,78 \%$ & $\begin{array}{c}\text { Gavarda (1.171), Antella (1.500), } \\
\text { Beneixida (636), Sumacàrcer } \\
(1.300), \text { La Pobla Llarga (4.451) }\end{array}$ \\
\hline
\end{tabular}


Una aproximación a la situación de los Agentes de Desarrollo Local (ADL) en el proceso de creación...

\begin{tabular}{|r|c|c|c|}
\hline MENOS DE 2 AÑOS & 3 & $16,67 \%$ & $\begin{array}{c}\text { Alginet (12.820), Senyera ( 1.045), } \\
\text { Benimodo (2.050) }\end{array}$ \\
\hline TOTALES & 18 & $100 \%$ & \\
\hline
\end{tabular}

Fuente- Elaboración propia

Habitualmente el técnico local de empleo aparece en un momento determinado fruto de la concertación de un convenio de colaboración entre la Corporación local y el organismo público -INEM en un primer momento y el órgano autonómico ${ }^{7}$ constituido al efecto después- a través de una subvención vinculada a la existencia de unos fondos económicos destinados al sufragio de un coste equivalente al $80 \%$-actualmente- de los salarios y la seguridad social de la contratación del técnico.

Este nacimiento ligado a la gratuidad de una subvención, supone que el motivo que origina la aparición de la agencia sea muy dispar, pero donde es destacable que una parte muy considerable de las Corporaciones ven en el técnico ADL una manera de disponer de un técnico cualificado subvencionado para el ayuntamiento, dado que con gran frecuencia exponen la limitación presupuestaria con la que cuentan, que se manifiesta en una carencia de personal (técnico o no).

Tabla 4. MOTIVO ORIGINARIO INICIOSDE LAACTIMDAD DE LA ADL

\begin{tabular}{|l|c|c|}
\hline \multicolumn{1}{|c|}{ MOTIVOS } & CANTIDAD & PORCENTAJE \\
\hline $\begin{array}{l}\text { DISPONER DE UN TECNICO CUALIFICADO } \\
\text { SUBVENCIONADO }\end{array}$ & 10 & $55,55 \%$ \\
\hline LA NECESIDAD DE DINAMIZAR EL MUNICIPIO & 3 & $16,67 \%$ \\
\hline LA CREACIÓN DE UN SERVICIO CON UN TÉCNICO & 2 & $11,11 \%$ \\
\hline SIN MOTIVO ESPECÍFICO & 3 & $16,67 \%$ \\
\hline TOTALES & 18 & $100 \%$ \\
\hline
\end{tabular}

Fuente.- Elaboración propia

\footnotetext{
${ }^{7}$ En el ámbito de la Comunitat Valenciana es el SERVEF (Servicio Valenciano para el Empleo y la Formación).
} 
Esta aparición de las agencias bajo una línea subvencionada, limita los recursos disponibles para la misma. En la tabla 5 podemos observar el número de recursos humanos disponibles por las agencias. En ella, destacamos nuevamente la importancia del número de habitantes que nos permite observar la evolución de las agencias de municipios de cierto tamaño hacia la constitución de un equipo multidisciplinar.

La limitación temporal de la subvención mencionada, máximo de cuatro años en la actualidad renovables de manera anual, está conduciendo a muchos municipios, sobretodo los más grandes, al inicio de un paulatino proceso de asunción directa -contratación- por parte del ayuntamiento. Aspecto que ha permitido abrir las puertas a una nueva contratación "subvencionada" de otro ADL en el municipio y así posibilitar la existencia de un equipo con perfiles distintos que cubran un abanico más amplio de actuaciones y programas para el empleo, tanto en cantidad como calidad de los mismos.

Tabla 5. RECURSOS HUMANOS DISPONIBLESEN LAS AGENCIAS

\begin{tabular}{|l|c|c|c|}
\hline & NÚ MERO & PORCENTAJE & $\begin{array}{c}\text { MUNICIPIOS Y NÚ MERO } \\
\text { DE HABITANTES }\end{array}$ \\
\hline ADL'S UNIPERSONALES & 13 & $72,23 \%$ & $\begin{array}{c}\text { Alberic (10.081), Alginet (12.820), Antella } \\
\text { (1.500), Beneixida (636), Benifaió (12.208), } \\
\text { Benimodo (2.050), Càrcer (2.040), Gavarda } \\
(1.171), \text { La Pobla Llarga (4.451), Senyera (1.045), } \\
\text { Sumacàrcer (1.300), Tous (1.116), Turís (5.556) }\end{array}$ \\
\hline ADL'sCON 2 MIEMBROS & 2 & $11,11 \%$ & L'Alcúdia (10.838), Algemesí (26.740), \\
\hline ADL'SCON 3 MIEMBROS & 2 & $11,11 \%$ & Carcaixent (21.299), Carlet (14.812) \\
\hline ADL'SCON 4 MIEMBROS 0 MÁS & 1 & $5,55 \%$ & Alzira (42.543), \\
\hline TOTALES & 18 & $100 \%$ & \\
\hline
\end{tabular}

Fuente- Elaboración propia

En las siguientes dos tablas -ver tablas 6 y 7 - presentamos informaciones referidas a los contenidos del trabajo del ADL. Concretamente se plantea una cuestión de fondo presente en su día a día, como es la amplitud funcional de la actividad del técnico, aspecto en el que nuevamente la variable independiente tamaño del municipio tiene su importancia. De hecho una dinámica habitual en municipios pequeños es por tanto la ampliación de las funciones es- 
tablecidas teóricamente para los técnicos, donde la variedad de actividades municipales desarrolladas por los ADL's también merece una mención, ya que suponen una sobrecarga -en muchos casos- determinante en el desarrollo de su actividad y que impide que la desarrolle plenamente.

\section{Tabda 6.}

\begin{tabular}{|l|c|}
\hline MUNICIPIOS PEQUEÑO $^{\mathbf{8}}$ & \\
\hline DESARROLLO LOCAL & $\mathbf{6 5} \%$ \\
\hline OTRAS TAREAS MUNICIPALES & $35 \%$ \\
\hline
\end{tabular}

\begin{tabular}{|l|c|}
\hline RESTO DE MUNICIPIOS & \\
\hline DESARROLLO LOCAL & $90 \%$ \\
\hline OTRAS TAREAS MUNICIPALES & $10 \%$ \\
\hline
\end{tabular}

Fuente- Elaboración propia

Talda 7. ACTIMDADES MUNICIPALESDESARROLLADAS POR LOSADL'S

\begin{tabular}{|l|c|}
\hline ACTIVIDADES MUNICIPALES & PORCENTAJE \\
\hline ALCALDIA & $1,11 \%$ \\
\hline AGRICULTURA & $1,11 \%$ \\
\hline ATENCIÓN AL CIUDADANO & $2,50 \%$ \\
\hline CULTURA & $4,72 \%$ \\
\hline COMERCIO & $4,44 \%$ \\
\hline DEPORTES & 0,83 \\
\hline FORMACIÓN Y OCUPACIÓN & $60,00 \%$ \\
\hline JUVENTUD & $1,94 \%$ \\
\hline MEDIO AMBIENTE & $0,83 \%$ \\
\hline PERSONAL & $2,22 \%$ \\
\hline PROMOCIÓN ECONÓMICA & $11,68 \%$ \\
\hline SERVICIOS SOCIALES & $1,67 \%$ \\
\hline TURISMO & $3,06 \%$ \\
\hline URBANISMO & $3,89 \%$ \\
\hline \multicolumn{1}{|c|}{ TOTAL } & $100 \%$ \\
\hline
\end{tabular}

Fuente- Elaboración propia

${ }^{8}$ Aunque no se trate de ninguna clasificación estricta, nos referimos a municipios de menos de 10.000 habitantes. 
Esta concepción ampliamente compartida e implantada del ADL como un técnico comodín que la Corporación local -recordemos de la que depende contractual y salarialmente- puede emplear en cualquiera de los menesteres locales, desemboca en una infravaloración por parte del resto de dependencias y servicios municipales del trabajo de un ADL -o de la Agencia en su conjunto. Valoración siempre muy por debajo de la importancia real de su labor. Esta depreciación profesional -muy vinculada con la inexistencia de un perfil profesional, una titulación académica de referencia o una descripción adecuada del puesto de trabajo- ha causado que los técnicos ADL's no participen mayoritariamente en los procesos de diseño y creación de los polígonos industriales. Esta afirmación hay que conectarla con la titulación profesional de los técnicos entrevistados -en su mayoría egresados en Derecho, Psicología y Relaciones Laborales- con poca experiencia previa en el desarrollo local y que acceden a un puesto de trabajo, el de ADL, sin conocer plenamente la labor a ejercer por el mismo y sin que nadie, en muchos de los casos, les establezca las directrices básicas de su actividad.

Ante esta situación, como la de desarrollo económico del territorio manifestado en la creación de suelo industrial, en el que el ADL por definición debería ser la pieza clave para la promoción y activación del desarrollo local, la realidad nos muestra cómo en su mayoría son apartados de este proceso. Se establece una jerarquía en la importancia de las funciones municipales desarrolladas, dándose clara prioridad a otras como urbanismo, alcaldía, ciudadanía o comercio.

\section{Tabla 8. MUNICIPIOS EN PROCESO DE CREACIÓN DE POLÍGONOS INDUSTRIALES}

\begin{tabular}{|l|c|c|}
\hline & CANTIDAD & PORCENTAJE \\
\hline $\begin{array}{l}\text { MUNICIPIOSEN PROCESO DE } \\
\text { CREACIÓN DE POLÍGONOS } \\
\text { INDUSTRIALES }\end{array}$ & 12 & $66,67 \%$ \\
\hline $\begin{array}{l}\text { MUNICIPIOS SIN PROCESO DE } \\
\text { CREACIÓN DE POLIGONOS } \\
\text { INDUSTRIALES }\end{array}$ & 6 & $33,33 \%$ \\
\hline TOTAL & 18 & $100 \%$ \\
\hline
\end{tabular}

Fuente- Elaboración propia 


\section{Tabla 9. PARTICIPACIÓN DE LOSAGENTES DE DESARROLLO LOCAL EN EL PROCESO DE CREACIÓN DE LOS POLÍGONOS INDUSTRIALES}

\begin{tabular}{|c|c|c|}
\hline & CANTIDAD & PORCENTAJE \\
\hline A. ADL'S CON PARTICIPACIÓN & 2 & $16,67 \%$ \\
\hline $\begin{array}{l}\text { A.I DIRECTA CON PODER DE } \\
\text { DECISIÓN }\end{array}$ & 1 & \\
\hline $\begin{array}{l}\text { A.2 INDIRECTA COMO UN } \\
\text { ORGANO SOCIAL MÁS DEL } \\
\text { MUNICIPIO }\end{array}$ & 1 & \\
\hline B. ADL'S SIN PARTICIPACIÓN & 10 & $83,33 \%$ \\
\hline TOTAL & 12 & $100 \%$ \\
\hline
\end{tabular}

Fuente- Elaboración propia

\section{CONCLUSIONES FINALES}

Es por tanto una situación compleja a la que se enfrentan habitualmente los técnicos ADL en el desarrollo de su actividad profesional, ya que siendo los responsables directos de la implementación de las actuaciones a nivel local para el empleo, no son tenidos en cuenta en procesos - como el que hemos expuesto de creación de suelo industrial- que están muy directamente vinculados con el mismo. Los ADL's podrían convertirse en el agente intermediario captador de estas empresas, entendidas como nuevas fuentes de empleo para la localidad. Además son los técnicos preparados para un diseño adecuado de las necesidades formativas de los futuros empleados, que cuentan con información sobre la situación del mercado de trabajo local, y que conocen de primera mano las características del territorio en cuestión.

En este sentido, tres son a nuestro entender las líneas principales de factores causantes que al respecto podemos apuntar: las características propias de la actividad, la competencia interna con otras concejalías municipales y la inexperiencia del modelo de desarrollo local.

En primer lugar, la insuficiente definición del puesto de trabajo, y en general de la actividad, suponen un considerable freno a las potencialidades de 
su labor. Características propias del trabajo del ADL no favorecen esta situación ya que el tipo de actividad y las características propias de la misma -plazos de ejecución ajustados, imposibilidad de una planificación adecuada, recursos limitados, dependencia de programas subvencionados.

En segundo lugar, la posible competencia interna entre concejalías interesadas en ser las encargadas de desarrollar esta labor tampoco favorece en nada, ya que se deja de lado el fin social colectivo pretendido por cualquier servicio público y se priorizan intereses ocultos de poder político. En este sentido la implicación del político responsable del área de empleo -o promoción económica- para con la actividad de la agencia es fundamental. Si el concejal asignado no es capaz de situar la actividad del técnico ADL al mismo nivel que el de otros técnicos locales difícilmente se conseguirá valorar su labor.

Y en tercer y último lugar, dada la juventud del modelo -aunque el modelo lo datemos en la década de los ochenta, recordemos que el grueso de técnicos aparece en la década de los noventa-, la manifiesta consideración de su trabajo como una actividad de carácter complementario, secundario o de segundo nivel en la ordenación de las funciones desarrolladas por la Corporación local, no ayudan de ninguna manera a su plena integración en el municipio, lo que origina una muy visible prevalencia de otras actividades consideradas como más importantes -es el caso de urbanismo, alcaldía y en algunos supuestos incluso servicios sociales, cultura, etc.- sobre ella.

\section{REFERENCIAS BIBLIOGRÁFICAS}

ALBURQUERQUE, F. (2002): Desarrollo económico territorial. Guía para agentes, Instituto de Desarrollo Regional. Sevilla. Fundación U niversitaria.

CACH ÓN, L. ( 1999): Sobre desarrollo local y nuevos yacimientos de empleo. Política y Sociedad, núm. 31. Madrid.

COMISIÓN DE LAS COMUNIDADES EUROPEAS (1993) : Libro blanco sobreel crecimiento, competitividad y empleo. Bruselas, COM (93) 700 final.

RODRÍGUEZ, F. (coord.) (1999): Manual de Desarrollo Local. Oviedo. CeCodet.

SANCHÍS, J. R (2000): M anual para agentes de inserción socio-laboral, Valencia, UNED. 
SANCHÍS, J. R. (2002): M anual para agentes de inserción socio-laboral: desarrollo local y creación de empresas. Valencia, UNED.

VÁZQUEZ BARQUERO, A (1988): Desarrollo local: una estrategia de creación de empleo. Madrid, Pirámide.

VACHON, B. (2001): El desarrollo territorial. T eoría y práctica. O viedo. CeCodet.

VV. AA. (1991) : M anual de desarrollo económico local. Madrid, FEMP. 